\title{
BendFlip: Examining Input Techniques for Electronic Book Readers with Flexible Form Factors
}

\author{
Doug Wightman, Tim Ginn, and Roel Vertegaal \\ Human Media Lab, Queen's University, Kingston, ON K7L 3N6, Canada \\ \{wightman, ginn, roel\}@ Cs. queensu.ca
}

\begin{abstract}
We present recommendations for the design of flexible electronic book readers, based on an empirical evaluation of form factors and input techniques in a page navigation task. We compared capacitive touch, pressure, and bend sensors between rigid and flexible form factors using a prototype electronic book reader. Results suggest that the time required to perform bend techniques is comparable to button techniques for page navigation on flexible form factors. We also found that a bend technique had fewer errors on flexible form factors. We discuss the physical affordances of flexible e-book form factors, and why they might be preferable to rigid designs. We conclude by presenting recommendations for the design of future flexible e-book readers.
\end{abstract}

Keywords: Flexible displays, electronic book readers, bimanual input.

\section{Introduction}

For some time, researchers have advocated for electronic book readers that preserve more of the physical affordances of paper documents [15,21,25]. According to O'Hara et al. [17], paper document navigation supports the development of incidental knowledge of the location of information within documents. Sheets of paper are lightweight displays that can be navigated by physically moving them. Sellen and Harper describe some of the disadvantages of Graphical User Interfaces (GUIs) [21]. When compared to paper documents, GUIs feature input that is indirect, often one-handed, and heavily dependent on visual as opposed to tactile cues. This can make the interleaving of multiple tasks across several displays cumbersome.

In recent years, the development of e-ink displays [6] has solved some of the above issues. Electronic ink displays feature a paper-like high contrast, and low power consumption, in a relatively lightweight form factor. However, most e-ink products still feature form factors that are much thicker and heavier than paper documents. The current generation of e-ink e-book readers, such as the Amazon Kindle [1], cannot yet be seen as a replacement technology for paper documents.

More recently, flexible display technologies have been developed (see Figure 1) [18]. These displays are compelling for future human-computer interaction design because they can provide more of the physical affordances of paper:

1. These displays are potentially as lightweight as paper. Users can hold these displays in one hand for extended periods of time without much effort. The Kindle DX, an e-ink e-book reader, by contrast, weighs more than a pound. 
2. These displays are potentially as thin as paper. One could stack multiple displays on a real-world desk, allowing for tighter interleaving of tasks across multiple displays, as is seen in interactions with paper [21].

3. Thin, flexible displays can feature input techniques that are similar to those used in paper documents. Flexible displays, like paper, bend. This capacity to bend provides an opportunity for explicit input $[19,20]$. Since bending affects the shape of the device, it provides tactile, kinesthetic as well as visual feedback.

4. Flexible displays are mobile. A flexible display can be deformed to fit inside a pocket. A large flexible display might be rolled up to fit in a backpack.

5. The screen real estate of a flexible display, like paper, can be easily varied. There are benefits to using a device that can have a large screen only part of the time. A paper road map can be opened for viewing large distances and then folded to provide a smaller form factor that contains all the information required for the next couple of hours of driving.

In this paper, we examine how we might provide input to future handheld flexible devices, such as e-book readers. We evaluated form factors and input techniques for flexible devices, with a focus on tasks found in e-book readers. We present an empirical evaluation of input techniques for the most frequently occurring task on ebook readers: single page navigation. In our experiment, we compared the use of capacitive touch, pressure sensors (buttons with varying force), and bend sensors for navigating single pages on an e-book reader with both rigid and flexible form factors. Results indicate that, given a flexible form factor, bends have comparable performance to buttons, and can have fewer errors. The performance of some input techniques is also notably comparable between rigid and flexible form factors. We conclude with recommendations for the design of future flexible e-book readers.

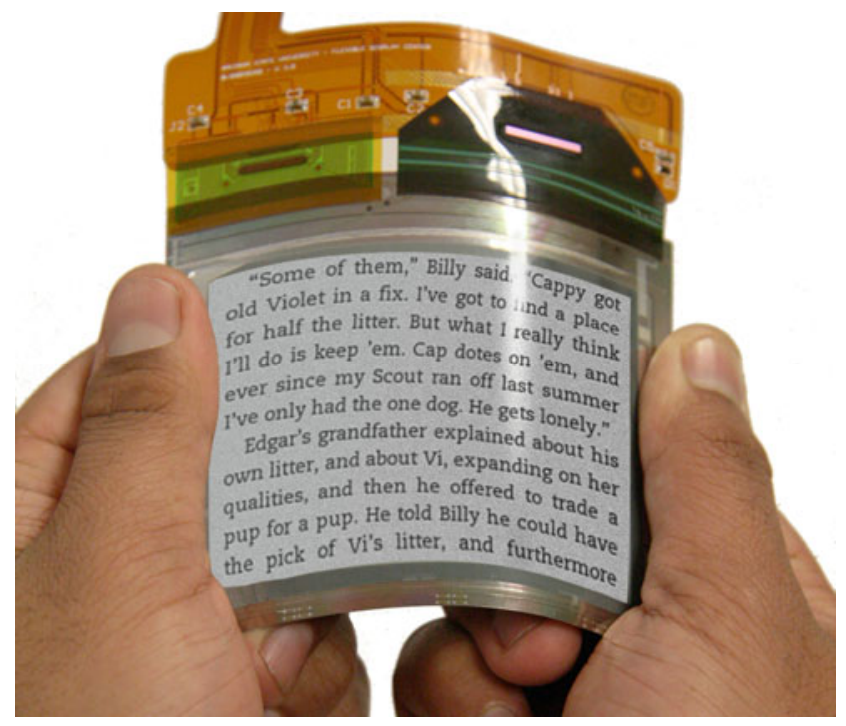

Fig. 1. E-ink Flexible Display 


\section{Related Work}

Balakrishnan et al. [2] discuss how they used ShapeTape, an optical bend sensor, to model curved surfaces on a computer. Flexible input techniques can operate in many dimensions. This makes it possible for users to twist and bend a device in ways that potentially activate more than one sensor at a time. They also warned of an "iron horse effect" when attempting to bring affordances of real world devices into digital systems: in designing a flexible electronic book reader it is important to copy only those properties of physical books that are desirable in some way, and to avoid unnecessarily copying the disadvantages of physical books.

TWEND was a hardware prototype developed by Herkenrath et al. [11] that allowed complex navigations using twists and bends. TWEND was constructed out of 8 optical bend sensors in order to be able to recognize a wide variety of contortions. While they did not provide an evaluation, they postulated that: "New users consider the page flipping and page scrolling gestures more efficient than techniques found in PDAs and mobile phones."

Lee et al. [13] conducted a user study of deformable materials. In their experiment, they asked users to create and perform gestures for 11 different tasks using thin plastic sheets, paper and an elastic cloth. They used paper to take advantage of untrained users examining what gestures they might find interesting for future flexible display input. They found a significant preference and time difference in how long users took to plan their gestures between cloth and plastic with elastic cloth having the highest preference and lowest planning time. They also found the left hand played mainly a supporting role, while the right hand performed most interactions on the middle of the right side of the material. Surprisingly, users were more consistent in the development of their gestures with materials that allowed more freedom, such as elastic cloth, than with materials that allowed less freedom, such as thin plastic.

Wobbrock et al. [27] emphasized the need for mobile devices without styli and proposed the use of back-of-device navigation. Amongst their findings are that the index finger on the back of the device is relatively worse for complex gestures than it is for simple moving and pointing tasks. Thumbs and index fingers performed similarly on the front of the device, but performance of thumbs decreased when moving to the back, while performance of index fingers did not. Additionally, their study showed that for one-handed and two-handed input, error rates mimicked movement time results. The best performance was achieved with the index finger on the front of the device. Some potential benefits of back-of-device navigation include not obscuring the screen or altering natural gripping or holding posture, and allowing users to grasp the bulk of the device rather than simply the lowest third of the device. Some of these benefits could apply to flexible electronic book readers.

Baudisch and Chu [3] investigated back-of-device navigation on very small touch devices. They found high accuracy across small screen sizes (2.4" and smaller), including in situations in which front of device navigation with a touch screen fails due to occlusion. They found that it was important for users to still be able to see part of their hand, so as to predict the location of their fingers on the back of the device. An electronic book reader could take advantage of this same property by having buttons or controls on the back of the device near the edges, where most of the hand remains visible. 
Wilson and Landoni [26] evaluated the page navigation features of four electronic book reader applications. Their design recommendations for future electronic book reader applications include simple bookmarking techniques that adhere to the paper book metaphor. Careful design of button and dial placement for page navigation was found to improve performance. In their evaluations, users of devices that employed dials commented that they felt they could read faster. Simple "page forward/page back" buttons were also considered intuitive, but button size mattered.

Harrison et al. [10] describe a user interface for document navigation applications that used pressure sensors in the top left and right corners of the display, to detect flicking gestures. It did not sense bend gestures. Paging through the document was achieved based on the direction and location of presses. Despite simulating the interaction technique of physical books, this technique is not necessarily the most efficient for page navigation. There are a couple of reasons for this: first, the movement required to complete the gesture takes more time than the theoretically most efficient technique, an in-place tap. Second, the pressure sensors they used required a certain amount of force to be triggered, which caused enough resistance for users to report they were not as lightweight as flicking through paper pages.

Chen et al. [5] discuss the use of flipping and fanning navigational techniques for electronic book readers. In their system, two small displays were joined together through a hinge, providing some of the physical affordances of a paper book. Their interface mimicked the turning of physical pages. Flipping was used for small navigations within the document (such as between pages) and fanning was used for large navigations (such as between chapters). However, real paper is extremely lightweight, making physical page turns rather effortless. Their techniques required gross motor movements to fold the entire hinged display. The authors indicated that they might have attempted to borrow "too literally" from book interactions, particularly given the size and weight of their device.

Gummi is one of the few projects to propose actual physical deformation of a display as a navigational technique. Schwesig et al. $[19,20]$ designed a mockup of a small credit-card sized computer. Navigation was achieved through bending the display, e.g., to zoom in and out of information. Similar in architecture to BendFlip, the interface was implemented using a rigid form factor display and a flexible sheet of acrylic, augmented with pressure sensors. Gummi was not designed as an electronic book reader and the authors did not report on its use for page navigation.

There is limited prior work on page navigation with flexible devices. In PaperWindows, Holman et al. [12] simulated the use of flexible display surfaces for page navigation by augmenting real paper with projected images. This was accomplished by tracking the shape and location of paper documents using computer vision. Pages could be navigated by flipping over a sheet of paper. This action required considerable gross motor activity, thus reducing its efficiency.

Similar in nature, Watanabe et al. [24] discuss Bookisheet, a set of flexible input devices made out of sheets of thin acrylic augmented with bend sensors. Bookisheet could simulate the turn of pages through bends. Bookisheet changes between discrete jumping and continuous scrolling modes based upon the degree of bend between the two pages that compose the device. Bookisheet also features "finger-bookmarking", which provides a temporary bookmark while a sensor is pressed. 
Pressure Sensors and Haptic Feedback. One issue with mobile display devices is that they are often used in environments such as busses or trains that are bumpy. Small buttons can be difficult to press in such environments. Brewster et al. [4] compared standard buttons to buttons with tactile feedback for a text entry task on a mobile device. Tactile feedback was found to increase the amount of text entered, reduce error rates (by $22.4 \%$ in the lab and $25.7 \%$ when mobile) and cause users to correct more errors (48.3\% in the lab, 66.9\% when mobile) compared to the same device without tactile feedback. Only rigid devices and input techniques were investigated. Luk et al. [14] investigated lateral skin stretch for mobile applications. Tactile feedback technologies, including lateral skin stretch hardware, could also be applied to flexible devices.
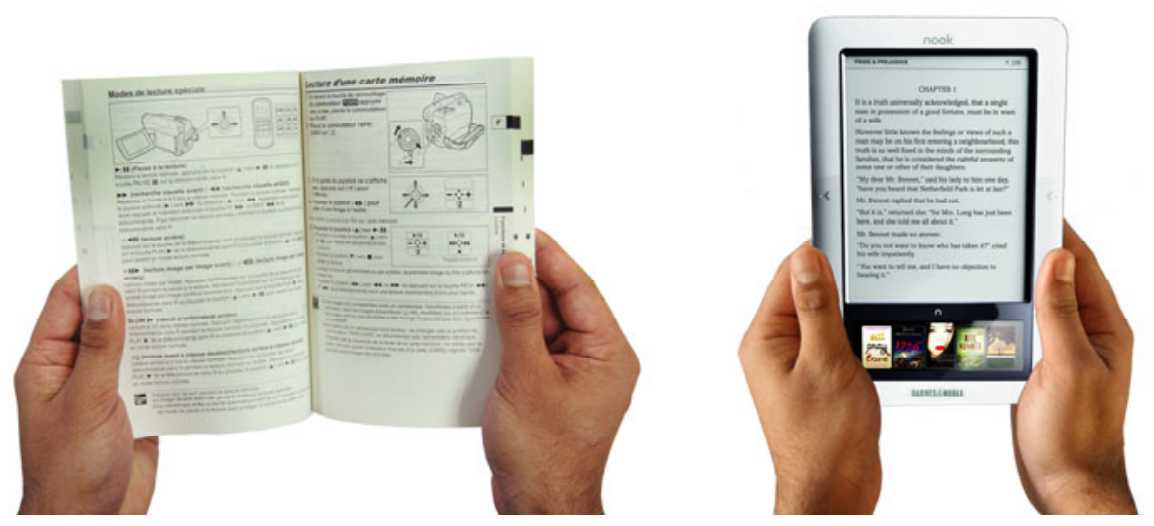

Fig. 2. Example hand positions when holding a paper book and e-book reader

\section{Design Rationale}

To educate ourselves on the requirements for the design of a flexible e-book reader, we informally observed individuals reading paper books as well as using two commercial e-book readers, the Amazon Kindle [1] and the Barnes and Noble Nook [16]. We were particularly interested to learn the ways in which users navigated single pages, which we found to be the single most frequently performed task while reading. In paper books, page turns are typically accomplished by turning the page through picking up the corner, less frequently by flicking the page up using the thumbs on the side of the page. Both gestures involve gross motor movements of the hand. By contrast, electronic book readers offer only buttons on their bezel for single page navigation. An advantage of these buttons is that they can be used to tap in place and do not require gross motor activity.

From our analysis of the literature and user observations we can identify a number of key design factors of e-book reader form factors that we believe are closely tied to the performance of flexible e-book reader designs. 


\subsection{Size and Portability}

Size, of course, is particularly relevant in mobile scenarios, where users should not only be able to carry the device, but also use it in potentially cramped seating; for example, during a rush-hour public transit commute. However, the requirement to be small impacts the ability to read comfortably. While small enough to fit inside a pocket, most smart phone form factors do not allow a comfortable reading experience due to their limited screen real estate. More screen real estate allows proper use of headlines to direct attention in large format newsprints. Just like paper, it appears, different size formats serve different functions. With its 10" screen, the Kindle DX is unsuitable for most mobile tasks, but allows for superior reading experiences with larger documents.

\subsection{Size and Ergonomic Fit}

Size is also relevant to the ergonomics of holding an e-book reader, and as such, to the placement of input sensors on the device. Figure 2 shows common hand positions for holding a book and an e-book reader with two hands. Fingers are often placed at the back of the display, with the thumbs at the front locking the device into the space between the thumb and index finger. Frequently used buttons on the front of the display should be placed in close proximity to the thumbs. There has been little experimentation in the commercial e-book market with buttons on the back of the display for paging [3]. It is unclear whether these could allow for more efficient movements than are possible with the thumb [27].

\subsection{Energy Expenditure}

Size also affects the efficiency of input methods. An input technique is more efficient when movement energy is minimized, given an accuracy criterion [8]. This means that, given an expert user and the task of paging forward one page, a button placed directly underneath the thumb is likely to be more efficient than a swipe gesture that requires movement of wrist and finger across the entire width of the screen. The Apple iPad e-book reader application borrows heavily from the paper book metaphor when it allows users to turn pages using a diagonal swipe gesture from the top right corner to the center of the display. While such gestures are beautiful, and facilitate learning transfer for users familiar only with paper books, they are also a good example of how too literal a use of analog book metaphors may introduce inefficiencies associated with the physics of paper books [5].

In one-handed scenarios, gestural input may be problematic when screens are larger than the range of an average thumb, approximately larger than an iPhone. If the non-dominant hand holds the device, the dominant hand is required for pointing, which increases the energy expenditure. Generally speaking, placement of frequently used buttons in locations that require a change of the natural grip produces an energy penalty to the user. Energy expenditure is particularly relevant to users reading books in a low-energy posture, such as while lying in bed. In such scenarios, books are often held up with one hand, or simply rested on a surface when they are heavy. The requirements for reading in bed are different from those in productivity-related reading scenarios. 


\subsection{Weight, Grasp and Force}

The size of the device impacts the center of mass relative to the hand holding the device [5]. The torque this produces is particularly relevant in one-handed scenarios: the further the center of mass is from the user's hand, the more force will be required to hold the device. This impacts the availability of the thumb to press a button or execute a touch screen gesture without losing grip. It also impacts the freedom necessary for a finger or thumb to rest on a button: the more normal force the device places on the thumb or fingers, the easier it is to press a button. This may produce spurious or unwanted actions, and may require users to rest their thumbs and fingers away from buttons.

Weight impacts fatigue: the heavier the device, the shorter the maximum time of unsupported use. This again is particularly relevant in more casual mobile and bed reading scenarios. With paper documents, we often observed users supporting the entire weight in a single skin crease, where the palm meets the fingers. Decreasing the weight of the device appears to benefit most usage scenarios.

\subsection{Grasp and Spurious Interactions}

Spurious interactions can be a problem with capacitive buttons and touch screens that overlap with locations where the device is often held. While we, in general, advocate the minimization of screen bezel size, one side effect of the presence of a significant bezel is that it allows the hand to hold the device without spurious interactions with a touch screen. The Kindle, Nook, iPhone, and iPad incorporate lock buttons that disable interactions during transport.

\subsection{Thickness and Rigidity}

Weight is dependent not only on the size, but also the thickness of the display. With the advent of thin-film flexible e-ink displays (Figure 1) it has become possible to consider usage scenarios in which an e-book reader is almost as thin and flexible as paper. Thickness of display also determines, along with the electronics and casing, the rigidity of the device, and as such is an important factor in the ergonomic fit of the display to the hand. While the Kindle features rectangular shapes that are somewhat uncomfortable to users, the Nook designers curved the back to allow users a more comfortable grip. By contrast, flexible displays such as the one in Figure 1 can automatically follow the shape of the hand. When holding a sheet of paper, we observed users bending the page slightly on a diagonal, starting from the lower left or right of the side of the page, so as to fit to their palm.

When screens are flexible, thinness does not only interact with weight, but also with display size and thus portability. Rollable e-ink technology introduced by Polymer Vision [18] allows screens to be rolled up when not in use, and extended when more screen real estate is required. Thinness also opens up different interaction spaces currently restricted to paper: the kind of interleaved multitasking scenarios described by Sellen and Harper [21]. These are possible only when displays are sufficiently thin and lightweight to be tossed around on a desk, or stacked in piles, without breaking the display [7]. 
Thinness and flexibility, however, may negatively impact the ability to interact with surface touch screens. Touch displays larger than a smart phone cannot be operated easily with one hand. Most require users to hold the display with the nondominant hand, typically on the left, freeing up the dominant hand for interactions with the surface of the display. However, a lack of support on one side of the display can cause it to bend when touched. This effect increases with screen width. One solution is to allow the dominant hand to continue to hold the display. However, this limits the extent of touch interactions, requiring users to rely largely on buttons placed along the bezel of the display for most frequent interactions, thus negating the need for a touch screen. An elegant solution often seen when operating a pen on paper is to seek the support of a rigid surface when needed.

One benefit of flexibility is that it opens up use of an alternate input channel that may be more robust to spurious interaction when handling the device: bend gestures. Paper document and book navigation often feature bending of a page as means of interaction. For example, when turning or bookmarking a page. Folding is a common means of scrolling content on paper maps. One potential benefit of bend sensors is that they allow interactions to remain the same when the display is folded. This is because bends can be detected relative to the current shape of the display, and can be detected relative to part of the surface using an array of bend sensors. By contrast, when a display is folded, physical buttons may be out of reach. We believe that, provided suitable solutions to some of the above problems are found, both thinness and the resulting flexibility of displays are desirable qualities.

\subsection{Readability and Responsiveness}

In the design of e-book readers, there are two further limiting factors that are unrelated to size, weight or thinness of the screen: contrast ratio/emissivity and refresh rate. For e-ink displays, these two relate. The use of polarized ink capsules in the display plane allows the simulation of a reflective paper display experience. The high contrast ratio caused by the lack of emissivity reduces fatigue. Reflectivity also improves battery energy consumption, an important factor in mobile technologies. However, at $150 \mathrm{~ms}$, the e-ink and flexible e-ink display refresh rate is slow.

\section{Flexible e-Reader Input Techniques}

We summarize our design space analysis as follows:

Size Matters. While a one-size-fits-all approach is suboptimal, the sizes of the Kindle and Nook e-book readers (about 8"x5"), are a reasonable reference for evaluating the design of a flexible e-book reader.

Optimize Movement and Ergonomic Fit. Frequently used functions on the front of the display should be placed in close proximity to common thumb positions when the device is held. Experimentation with back of the display interactions might be promising.

Button Forces Matter. Most buttons on rigid devices act as force sensors. On a flexible device, the force required to activate a button may deform the device. One 
means of measuring this force is to measure the extent of that deformation. Capacitive touch and buttons that require minimal force to trigger may increase error, while buttons that require a large force may never be actuated due to a lack of a sufficient normal force on flexible displays.

Flexible Displays Bend on Force. Experimentation with bending the display to perform frequently used functions appears promising. In one-handed scenarios, pointing with the dominant hand may be ineffective due to a lack of a normal force. Spurious Interactions Are a Source of Error. Capacitive, and other minimal force interactions, can increase the number of errors in actuation.

Display Weight Matters. Even a light device will cause fatigue during extended use. Screen Response Time Matters. The refresh rate of e-ink may be a confounding variable, particularly for existing page animation techniques.

\subsection{Focusing Design on Input for Most Frequent Task}

To focus our design and experimentation, we restrict our evaluation to input techniques that might be most suited for paging forward and backward through a document on a flexible e-reader form factor. Of the discussed options, we chose five representative input techniques for evaluation: 1) capacitive touch sensors; 2) pressure sensors (buttons) on the front of the display with varying forces; 3) pressure sensors (buttons) on the back of the display, also with varying forces; 4) pressure sensors with one-handed interactions and 5) bending gestures on the side of the display.

We limited our exploration of intentional bending to page flicking. These gestures require less physical effort than most bending gestures. Due to the limited variance in finger movement, we consider them to be most comparable to in place tapping. We further limited our focus to the efficiency and accuracy of interactions with the device. We note that this did not allow us to study calm or mobile aspects of the presented scenarios, and consider these beyond the scope of this particular study.
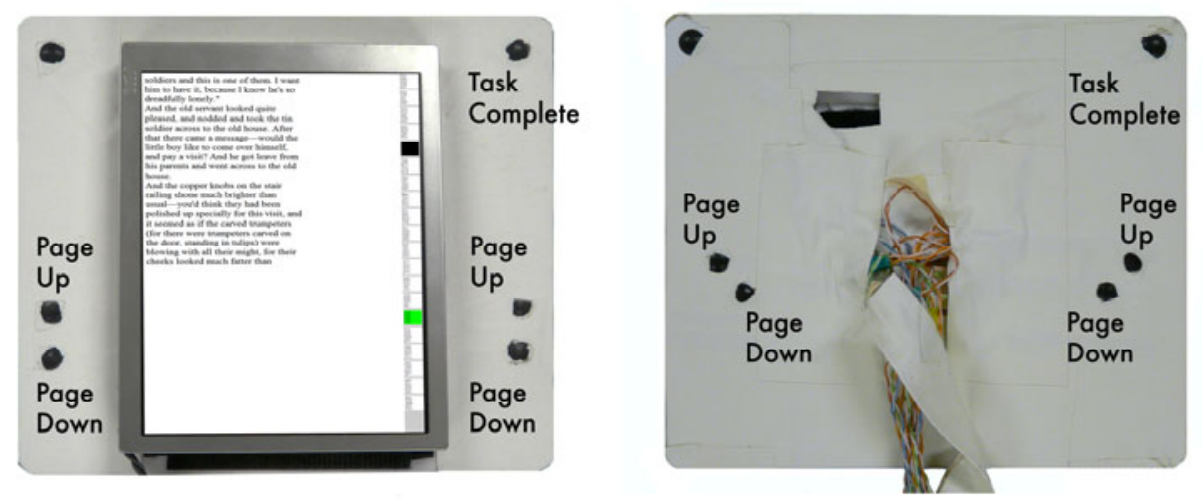

Fig. 3. Front and back of the BendFlip Experimental Apparatus 


\section{BendFlip: A Flexible e-Reading Apparatus}

Figure 3 shows BendFlip, an e-reader that merges properties of future flexible devices with the display refresh rate of a lightweight Kindle-size LCD screen. It served as our experimental apparatus. BendFlip consists of an 8 "x7"x1/32" thin, flexible surface outfitted with sensor inputs and a 200-gram rigid LCD display. We used an LCD display because the refresh rates on currently available flexible displays are too slow for our experiment (>150 ms). A 1/8" removable rigid backing, weighing 90 grams, was added to allow comparison of our set of inputs with a rigid e-book reader design. Figure 3 shows how capacitive touch, pressure, and bi-directional bend sensors were mounted on the front and back of the device. Sensor locations were selected based upon the ISO/TR 7250 standard finger measurements (70mm for index fingers). The pressure sensors created $2 \mathrm{~mm}$ bumps on the surface, which was otherwise taped in bookbinding tape to give it a paper feel. This bump allowed participants to locate the pressure sensors on the back of the device without looking. While pressure sensors do not provide a button click, tap performance is governed by trigger force, and feedback of page turns is displayed. A conductive cloth connected to a capacitive touch sensor covered each of the front pressure sensors. This allowed for capacitive touches to be detected on each of the black pressure sensors shown in Figure 3. Figure 4 shows a user holding the device with fingers on the sensors.

\subsection{Detecting Bend Gestures}

We used two bi-directional bend sensors to detect bends: one in the center of both the left and right sides of the device. On the right of Figure 4 we see a user bending a side of the device downwards. After several iterations of our initial bend detection algorithm, even small bends can be detected.

Our first algorithm required a 30-degree bend. The size of the bend was selected to differentiate bend from force input. We quickly realized that sensing bend over a smaller area, specifically the inch along the edge of the device, creates a different signal pattern than force input, which distributes the bend over a larger area. This design revision still detected bend using an absolute sensor value threshold.

After several trials with the revised design, we observed that our algorithm still failed to detect certain subtle flicks. For example, many participants did not allow the
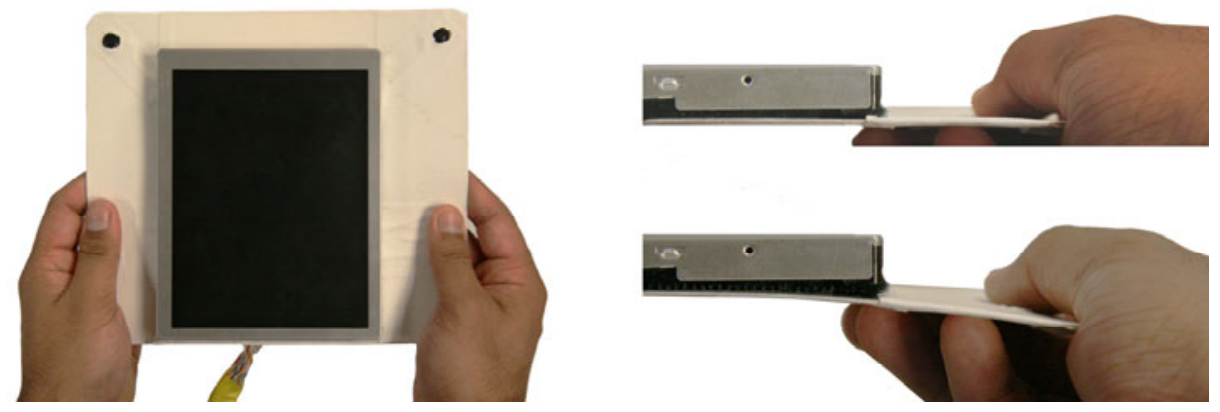

Fig. 4. Pressure and bend view 
device to return to its resting state in between flicks. In fact, many pilot participants preferred to hold the device in a bent state while flicking. To address this, we adjusted our algorithm to detect flicks based upon relative changes in the sensor readings. Our final algorithm creates upwards (or downwards) flick events when the edge of the device is bent at least slightly upwards (or downwards) after a peak deformation occurring within a window of one second.

\section{Evaluation}

We evaluated the following set of input techniques for single page forward and backward navigation with BendFlip. These techniques are intended to represent a range of alternatives found in the current generation of e-book readers, extrapolated to future devices:

1) Front Thumb Pressure (“front press"). The user's left and right thumbs are used to press the pressure sensors on the front of the device. The use of a pressure sensor rather than a button allowed for us to set the force required for actuation, at three levels: minimum, medium, and maximum.

2) Front Finger Pressure ("point"). The user holds the device with one hand, typically their non-dominant hand. The index finger on the user's dominant hand is used to activate the same sensors deployed in the Front Thumb Pressure condition, again with three different forces.

3) Back Finger Pressure ("back press"). The user's left and right hand index and middle fingers are used to actuate the pressure sensors on the back of the device.

4) Front Capacitive ("front capacitive"). Same as Front Thumb Pressure, except the pressure sensors are replaced with capacitive touch sensors that require minimal force for actuation.

5) Bend ("bend"). The user slightly bends the left or right side of the device down with the thumb or up with the back-of-the-device fingers.

23 different variations on each of these input techniques were evaluated experimentally. 6 variations of Front Thumb Pressure were evaluated: three different force settings, and with or without the rigid surface mounted behind the device. The three force settings were: $0.2 \mathrm{~N}$, the minimum amount that reliably activates the sensor ("min force"), 1.5 $\mathrm{N}$, approximately the force required to press the Next Page button on the Kindle 2 ("med force"), and $5.9 \mathrm{~N}$, the force required to bend the flexible form factor 40 degrees ("max force"). Front Finger Pressure and Back Finger Pressure were evaluated with these same 6 variations. Front Capacitive was evaluated with and without the rigid surface.

We also evaluated 3 different variations of bend gestures. In the first two, only the right side of the device is actuated:

1) Bend Right Side Only. Page up (navigating back) is triggered by a flick up, toward the user, and page down (navigating forward) by a flick down, away from the user. 
2) Bend Right Side Only Inverted. The page up and page down actions are reversed from Bend Right Side Only.

3) Bend Split Both Sides. In the third variation, both sides of the device are used. A flick down, away from the user on the left side of the device triggers a page up (navigating back), while a flick down, away from the user on the right side of the device triggers a page down (navigating forward). A second factor in our experiment was the rigidity of the device. In these conditions, a thin but rigid surface was mounted on the back of the device.

\subsection{Task, Design and Procedure}

Participants were asked to navigate between the current page and a target page in the same document. The current page appeared on the left side of the display, with a row of thumbnails on the right side of the display. The thumbnail for the current page was always colored in black. The document was twenty pages in length. Participants navigated back and forth between page 5 and page 15 in the document, 8 times for each of the 23 input techniques. This allowed participants to overshoot their targets within the document. At the beginning of each trial, which constituted navigating to one target, the target page thumbnail was highlighted in green (see Figure 3). A trial was completed once the participant had navigated to the target and pressed a pressure sensor on the top right of the device (marked "Task Complete" in Figure 3), indicating the end of that trial. The time to reach the target (the Seek Time), not the time to press the "Task Complete" pressure sensor, was used to calculate our results. Participants were trained to use each of the input techniques. Training consisted of a demonstration followed by practice. Participants completed training trials until their seek time improved by less than $10 \%$. There were 12 participants ( 7 male, 5 female), aged 20 to 26.11 of the 12 participants were right-handed.

The experiment design was a $7 \times 2 \times 2 \times 3$ repeated measures with four factors: input technique (front capacitive, front press, back press, bend right side only, bend right side inverted, bend split both sides, and point), form factor (flexible vs. rigid), input side (front vs. back) and pressure setting (min force, med force, max force). These factors are derived from the design rationale, specifically: ergonomic fit, rigidity, grasp, and spurious interactions.

All conditions were counterbalanced using a random order of presentation. The design was not fully factorial, in that bend was only implemented in the flexible form factor, and pressure settings only applied to pressure sensor input techniques. We performed two separate repeated measures analyses of variance (ANOVA). The first compared all input techniques exhaustively using Bonferroni corrected multiple comparisons. The second examined only effects between the fastest pressure input techniques, comparing front vs. back and rigid vs. flexible via a fully factorial design. The dependent measure was seek time in all cases.

We expected rigid and capacitive touch input techniques to be faster than the flexible input techniques. We also expected the bend input techniques would be slower than the fastest flexible input techniques.

To examine error, we also measured the number of page navigations required to complete each trial. If the number of page navigations was more than 10 , this 


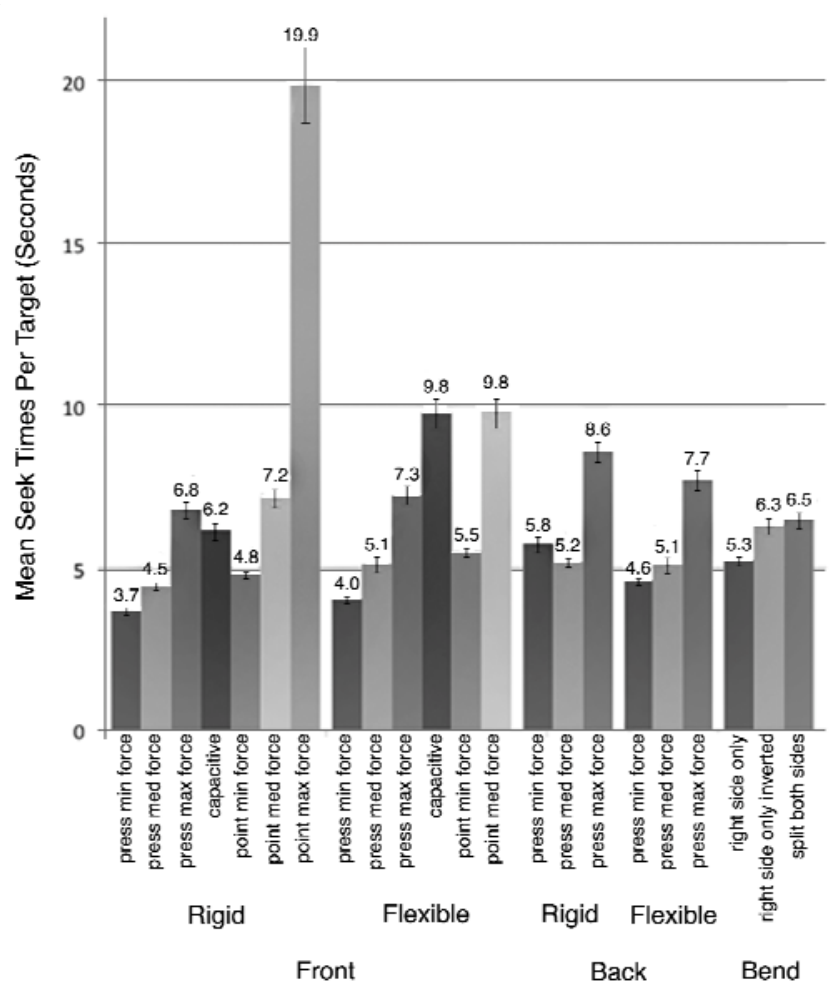

Fig. 5. Mean seek times for input techniques

constituted an error. Overshooting the target or accidentally navigating in the wrong direction would cause such errors. To evaluate performance on errors, we used an ANOVA with one factor: input technique. In this case, the dependent measure was the number of page navigations used to reach the target. To gauge their subjective opinions, participants completed a Likert-style questionnaire after each set of 8 trials.

\section{Results}

Two of the twelve participants were unable to complete the point flexible med force condition. These participants indicated that it was too difficult to activate the pressure sensors. None of the participants were able to complete the point flexible max force condition. We also removed the three slowest input techniques from analysis due to their unequal variances: front capacitive flexible (9.8 seconds, standard error 0.5), point flexible med force (9.8 s, s.e. 0.6), and point rigid max force (20.0 s, s.e. 1.3).

Figure 5 shows the results for mean seek time in seconds for each input technique. Analysis of variance showed a significant main effect for input technique: $\mathrm{F}_{18,247}=$ $5.7, \mathrm{p}<0.01$. The mean seek time for front press rigid min force was the lowest at 3.7 seconds (s.e. 0.1), followed by front press flexible min force (4.0 s, s.e. 0.2). A posthoc Bonferroni found significance between the fastest two input techniques (front 
press rigid min force and front press flexible min force) and each of front press rigid max force, front press flexible max force, back press flexible max force, and point rigid med force $(\mathrm{p}<0.05)$.

We also performed a fully factorial ANOVA to compare differences in performance between flexible and rigid form factors, as well as between input sides: front vs. back. We chose the fastest technique that minimized error for this comparison: min press. The main effect for input side was significant, with input on the front significantly faster than input on the back $\left(\mathrm{F}_{1,48}=9.9, \mathrm{p}<0.01\right)$.

\subsection{Errors}

On average, there were 11.0 page navigations for each trial (s.e. 0.25 ), while each trial required only 10 page navigations. Back press rigid min force had the largest number of page navigation errors. Back press flexible min force reported the second largest error (10.9, s.e. 0.28), followed by bend right only inverted (10.6, s.e. 0.14), front press flexible min force (10.5, s.e. 0.23$)$, and front capacitive flexible (10.5, s.e. 0.13 ). All other input techniques had error rates below $3.5 \%$. There was a significant main effect for input technique on error $\left(\mathrm{F}_{21,262}=3.7, \mathrm{p}<0.05\right)$. Multiple comparisons showed significant differences existed between input techniques with an error rate below 5\% and the two poorest techniques: back press rigid min force and back press flexible min force ( $\mathrm{p}<0.05$ in all cases, Bonferroni corrected).

\subsection{Qualitative Responses}

Mean responses to the 5-point Likert scale question "I enjoyed using this device" (1Strongly Disagree, 5-Strongly Agree) ranged from 1 (s.e. 0.0) for front press flexible max force to 4.75 (s.e. 0.13) for front press rigid min force. Bend right side only had a mean response of 4.08 (s.e. 0.26), comparable to back press flexible min force 4.08 (s.e. 0.29). We tested significance using a Friedman's Two-Way Analysis of Variance by Ranks on the Likert-scale enjoyment scores. The main effect was significant between input conditions (Friedman's $\chi 2=157.1, \mathrm{p}<0.01$ ).

\section{Discussion}

We believe that the unexpected poor performance of capacitive input on the flexible form factor was partially due to participants exercising caution to prevent spurious interactions. Possibly for the same reason, pressure sensors on the front of the display significantly outperformed those on the back of the display. As expected, holding the device with one hand and actuating a pressure sensor by pointing with the other hand was much slower and more error prone than holding the device with two hands.

The fastest input techniques for single page navigation have comparable seek times on flexible and rigid form factors. Flexible devices also have the added advantages of being lighter, more portable, and supporting bend input.

Bend right side only performed better than expected; with seek time and Likert enjoyment ratings comparable to the other flexible input techniques. Unlike bend right side only, the error rate for the fastest input technique on the flexible form 
factor, press med force, was above 5\%. Bend input can also easily be performed anywhere on the device, almost regardless of hand position, and without looking.

Questionnaire comments indicated that participants found bend right side only to be the most intuitive of the bend alternatives, and most similar to reading a paper book. Responses to the Likert scale enjoyment question for bend right side only were also favorable.

\subsection{Design Recommendations}

Our results are summarized in the following design recommendations for input on flexible e-book readers:

1. Single page navigation performance should not be the deciding factor when choosing between flexible and rigid e-book reader designs. Seek times for the fastest input techniques on the flexible and rigid form factors are comparable.

2. For flexible designs, bend is an excellent alternative to buttons. Bend input can have comparable performance and fewer errors.

3. When using bend to navigate pages, a single bend sensor on the dominant side of the device can be effective. Page forward should be triggered by bending the edge down, and page back by bending the edge up. Bending up to page forward and down to page up is not recommended. While one might consider placing a bend sensor on both sides of the flexible display to account for left-handed users, using one side to page back and the other to page forward is not recommended.

4. When using bend, ensure that bends are measured relative to the current shape of the display surface so as to avoid spurious triggering. For close to button performance, also minimize the extent of the bend.

5. Capacitive touch and minimal force buttons may increase spurious interactions on flexible designs when placed on the back of the device. This may be partially due to user reliance on haptic feedback. Capacitive touch is not recommended for flexible designs.

6. Buttons on the back of the device increase error. We believe this may be due to users not having visual feedback on their placement.

7. When using buttons, force settings close to $1.5 \mathrm{~N}$ appear optimal to avoid spurious interactions. Our medium force setting allowed participants to support the device and tap in place at the same time. This is currently the case with rigid e-book readers, and transfers to flexible designs.

8. When using buttons, they should be placed on the front edge of the device and underneath the thumbs to support tap in place operations.

Note that the use of pressure sensors does not preclude the use of bend sensors. The two can be combined in order to increase the number of available functions.

\section{Conclusions}

In this paper, we examined input techniques for future handheld flexible display devices. We presented an empirical evaluation of input techniques for the most frequently performed e-book reader task: single page navigation. In our experiment, 
we compared the use of capacitive touch, pressure sensors (buttons with varying force), and bend sensors for navigating single pages on an e-book reader with both rigid and flexible form factors.

We found that our most efficient bend technique had comparable performance to the button input techniques for the flexible form factor, and fewer errors. For this reason and others, bend may be a compelling alternative to capacitive touch and pressure sensor input. Results also suggest that flexible devices can have comparable performance to rigid devices for e-book reader tasks.

\section{References}

1. Amazon, Inc. Kindle Wireless Reading Device, http: / /www. kindle.com/

2. Balakrishnan, R., Fitzmaurice, G., Kurtenbach, G., Singh, K.: Exploring interactive curve and surface manipulation using a bend and twist sensitive input strip. In: Proc. I3D 1999, pp. 111-118. ACM Press, New York (1999)

3. Baudisch, P., Chu, G.: Back-of-device interaction allows creating very small touch devices. In: Proc. CHI 2009, pp. 1923-1932. ACM Press, New York (2009)

4. Brewster, S., Chohan, F., Brown, L.: Tactile feed-back for mobile interactions. In: Proc. CHI 2007, pp. 159-162. ACM Press, New York (2007)

5. Chen, N., Guimbretiere, F., Dixon, M., Lewis, C., Agrawala, M.: Navigation techniques for dual-display e-book readers. In: Proc. CHI 2008, pp. 1779-1788. ACM Press, New York (2008)

6. E-Ink, http://www. eink.com/

7. Engadget,

http: //www. engadget.com/2009/10/16/

please-hammer-dont-hurt-samsungs-flexible-oled-prototype-vid/

8. Fitts, P.M.: The information capacity of the human motor system in controlling the amplitude of movement. Journal of Experimental Psychology 47, 381-391 (1954)

9. Gallant, D.T., Seniuk, A.G., Vertegaal, R.: Towards more paper-like input: flexible input devices for foldable interaction styles. In: Proc. UIST 2008, pp. 283-286. ACM Press, New York (2008)

10. Harrison, B.L., Fishkin, K.P., Gujar, A., Mochon, C., Want, R.: Squeeze me, hold me, tilt me! An explora-tion of manipulative user interfaces. In: Proc. CHI 1998, pp. 17-24. ACM Press, New York (1998)

11. Herkenrath, G., Karrer, T., Borchers, J.: Twend: twisting and bending as new interaction gesture in mobile devices. In: Ext. Abstracts CHI 2008, pp. 3819-3824. ACM Press, New York (2008)

12. Holman, D., Vertegaal, R., Altosaar, M.: PaperWindows: Interaction Techniques for Digital Paper. In: Proc. CHI 2005, pp. 591-599. ACM Press, New York (2005)

13. Lee, S., Kim, S., Jin, B., Choi, E., Kim, B., Jia, X., Kim, D., Lee, K.: How Users Manipulate Deformable Dis-plays as Input Devices. In: Proc. CHI 2010, pp. 1647-1656. ACM Press, New York (2010)

14. Luk, J., Pasquero, J., Little, S., MacLean, K., Levesque, V., Hayward, V.: A role for haptics in mobile interaction: initial design using a handheld tactile display prototype. In: Proc. CHI 2006, pp. 171-180. ACM Press, New York (2006)

15. Marshall, C.C., Bly, S.: Turning the page on navigation. In: Proc. JCDL 2005, pp. 225-234. ACM Press, New York (2005)

16. Nook, http: / /www. barnesandnoble.com/nook/

17. O'Hara, K., Sellen, A.: A comparison of reading pa-per and on-line documents. In: Proc. CHI 1997, pp. 335-342. ACM Press, New York (1997) 
18. Polymer Vision, http: / / www . polymervision.com/

19. Schwesig, C., Poupyrev, I., Mori, E.: Gummi: a bendable computer. In: Proc. CHI 2004, pp. 263-270. ACM Press, New York (2004)

20. Schwesig, C., Poupyrev, I., Mori, E.: Gummi: user interface for deformable computers. In: Ext. Abstracts CHI 2003, pp. 954-955. ACM Press, New York (2003)

21. Sellen, A.J., Harper, R.H.R.: The Myth of the Paperless Office. MIT Press, Cambridge (2003)

22. Tajika, T., Yonezawa, T., Mitsunaga, N.: Intuitive page-turning interface of e-books on flexible e-paper based on user studies. In: Proc. MM 2008, pp. 793-796. ACM Press, New York (2008)

23. Universal Display, http: / / www . universaldisplay.com/

24. Watanabe, J., Mochizuki, A., Horry, Y.: Bookisheet: bendable device for browsing content using the metaphor of leafing through the pages. In: Proc. UbiComp 2008, pp. 360-369. ACM Press, New York (2008)

25. Wellner, P.: Interacting with paper on the DigitalDesk. Commun. ACM 36(7), 87-96 (1993)

26. Wilson, R., Landoni, M.: Evaluating the usability of portable electronic books. In: SAC 2003, pp. 564-568. ACM Press, New York (2003)

27. Wobbrock, J.O., Myers, B.A., Aung, H.H.: The performance of hand postures in front-and back-of-device interaction for mobile computing. Int. J. Hum. Comput. Stud. 66(12), 857-875 (2008)

28. Zhai, S., Kristensson, P.: Shorthand writing on stylus keyboard. In: Proc. CHI 2003, pp. 97-104. ACM Press, New York (2003) 